\title{
Design of Rolling Machine Intelligent Control System for Greenhouse Based on Internet of Things
}

\author{
Hongjun Gu a , Ji LI, Shijun LI ${ }^{b}$, He Gong, Jian LI, Lixin Hou \\ Jilin Agricultural University, Changchun 130118, Jilin, China \\ aghj_dennis@163.com, blsj0883@sina.com
}

\begin{abstract}
This paper designs a new rolling machine intelligent control system for greenhouse based on Internet of things (IOT). This system consists of ZigBee module, light sensor, controlled circuit, remote control unit, mobile, computer, AC contactor, limit switch, display circuit and keyboard. According to the general information of the time, light intensity outside, temperature and vegetable kind in the greenhouse and so on, ZigBee module decide whether the rolling machine should be opened and how many angles for open. Users can also operate it manually or remotely. The system realizes the intellegent control of roll blind machine for greenhouse. After a long work verification, the system has the performances of safety and reliability, low cost and can be embedded into agricultural IOT.
\end{abstract}

Keywords: IOT; Greenhouse; Rolling machine; Intelligent control.

\section{Introduction}

In recent years, the IOT technology has been gradually playing an important role in the field of agriculture. To improve the production and quality of agricultural products, and save manpower and material resources, modern information technology is used to guide agricultural production, which is the agriculture development direction. China has made significant achievements in agricultural greenhouse engineering facilities, high-yield cultivation and quality safety production technology. Modern greenhouse technology mainly uses the automation equipment to adjust the crop growth environment according to the collected factors, such as temperature, humidity, and light intensity, etc.

In traditional agriculture, farmers can only rely on intuition to control the rolling machine. With the development of science and technology, modern agriculture is developing towards the intelligent direction [3]. Agricultural IOT can solve the problems, such as whether fruits and vegetables needs watering, fertilizing, spray insecticide, and how to ensure the temperature, moisture, light, $\mathrm{CO}_{2}$ concentration of the greenhouse[4]. Modern intelligent monitoring and control system can improve the growth environment for the crops [5]. Of course, farmers can also manually operate the system via mobiles, computers, buttons and keyboard.

Rolling machine is used to keep the stable temperature and suitable humidity for the vegetables and fruits in the greenhouse. Introducing the new technology and advanced equipment is beneficial to improve the environment and save labour [6]. This paper presents a new rolling machine control system which can intelligently send the useful related data to the users and accept the control commands.

\section{Overall System Framework}

The intelligent control system uses ZigBee module as the core, which can communicate useful information with mobiles and computers, and send the commands to the controlled circuit of the rolling machine. The remote controller can realize the near distance wireless control of the machine. At the same time, manual switches can also change the working state of the machine. The overall system framework is shown in Fig. 1.

ZigBee module acquires the data from the light intensity, air temperature and humidity and sends them to the computer with Internet access via the gateway. The computer sends the data to intelligent mobile. The administrators can inquire the data from sensors and send control commands to the 
rolling machine via the mobile or the computer. The computer can analyze the gathered information and prepare for agricultural big data. The farmers can modify the pre-set air temperature and humidity via the mobile, the computer or the keyboard. The displaying screen can show the current time and date, pre-set parameters and collecting sensor information of many nodes. Several important parts are introduced in the following.



Fig. 1 The overall system framework

\section{Zigbee Minimum System}

The CC2530 is chosen as the control core. It is a true system-on-chip solution for IEEE 802.15.4, Zigbee and RF4CE applications. The CC2530 combines the excellent performance of a leading RF transceiver with an industry-standard enhanced $8051 \mathrm{MCU}$, in-system programmable flash memory, 8-KB RAM, and many other powerful features. This system adopts CC2530F256 with $256 \mathrm{~KB}$ of flash memory. The detailed minimum system diagram is shown in Fig .2.

This chip can collect the light intensity, air temperature and humidity, compare them with the pre-set values and consider time and date, decide whether the rolling machine should be opened and how many angles for open. Zigbee has ad-hoc network function, so the moisture data can be sent between nodes, and at last the related information can be accepted by the computer and mobile with the help of gateway.

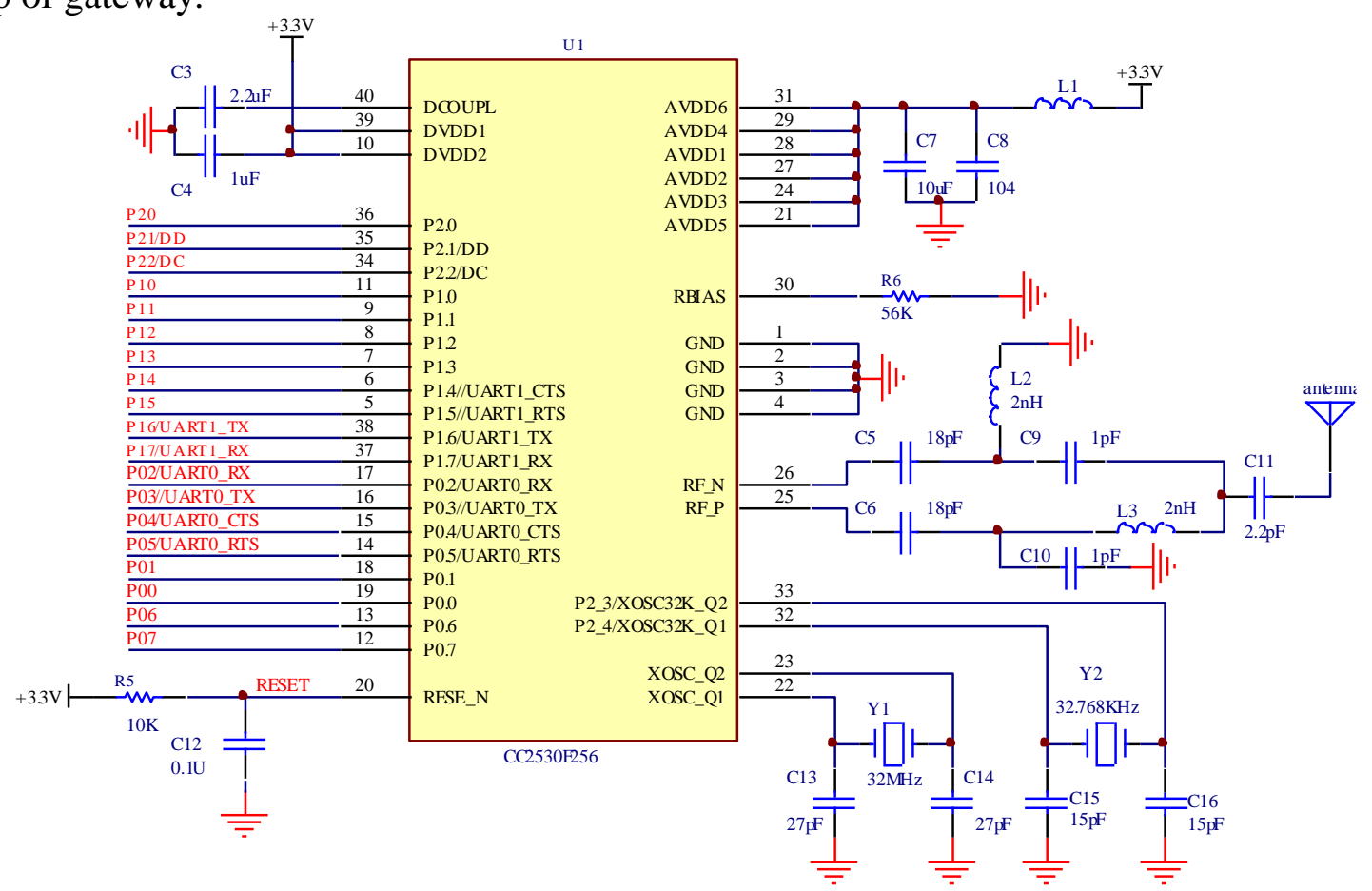

Fig. 2 CC2530 schematic diagram 


\section{Remote Control Unit}

Remote control unit uses PT2262/HS2272 to design the wireless transmitting and receiving modules, respectively, and the products are shown in Fig. 3. The communication distance of the device with the operating voltage range $3-12 \mathrm{~V}$ can reach above 1000 meters, which can satisfy the application in the greenhouse.

The transmitter has 3 keys representing for the commands 'up/down/stop'. The receiver gains the corresponding information to operate the rolling machine. The users can control the machine at the entrance without entering the greenhouse.

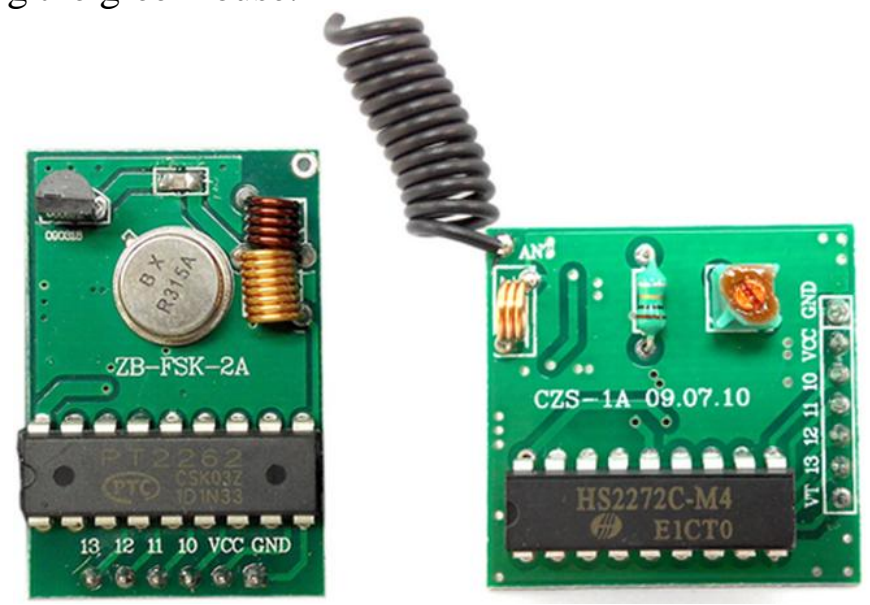

Fig. 3 Products of remote control unit

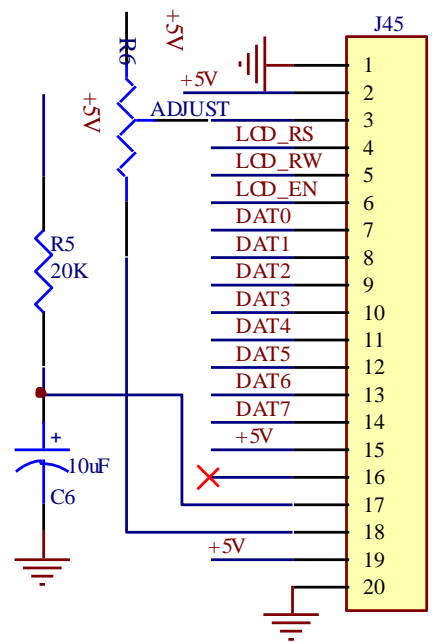

Fig. 4 Displaying circuit schematic diagram

\section{Displaying Circuit Design}

Displaying circuit adopts LCD12864 which can show the current time and date, light intensity, air temperature and humidity values of many nodes and the states of the rolling machine. As shown in Fig. 4, LCD12864 with 20 pins communicates with CC2530 by DAT0--DAT7, LCD_RS, LCD_RW, and LCD_EN. DAT0--DAT7 means transmitting data or commands. LCD_RS decides DAT0--DAT7 representing data or commands. LCD_RW means 'read' or ,'write' operation. LCD_EN gets a pulse, and data or commands have been writen in the LCD12864. 


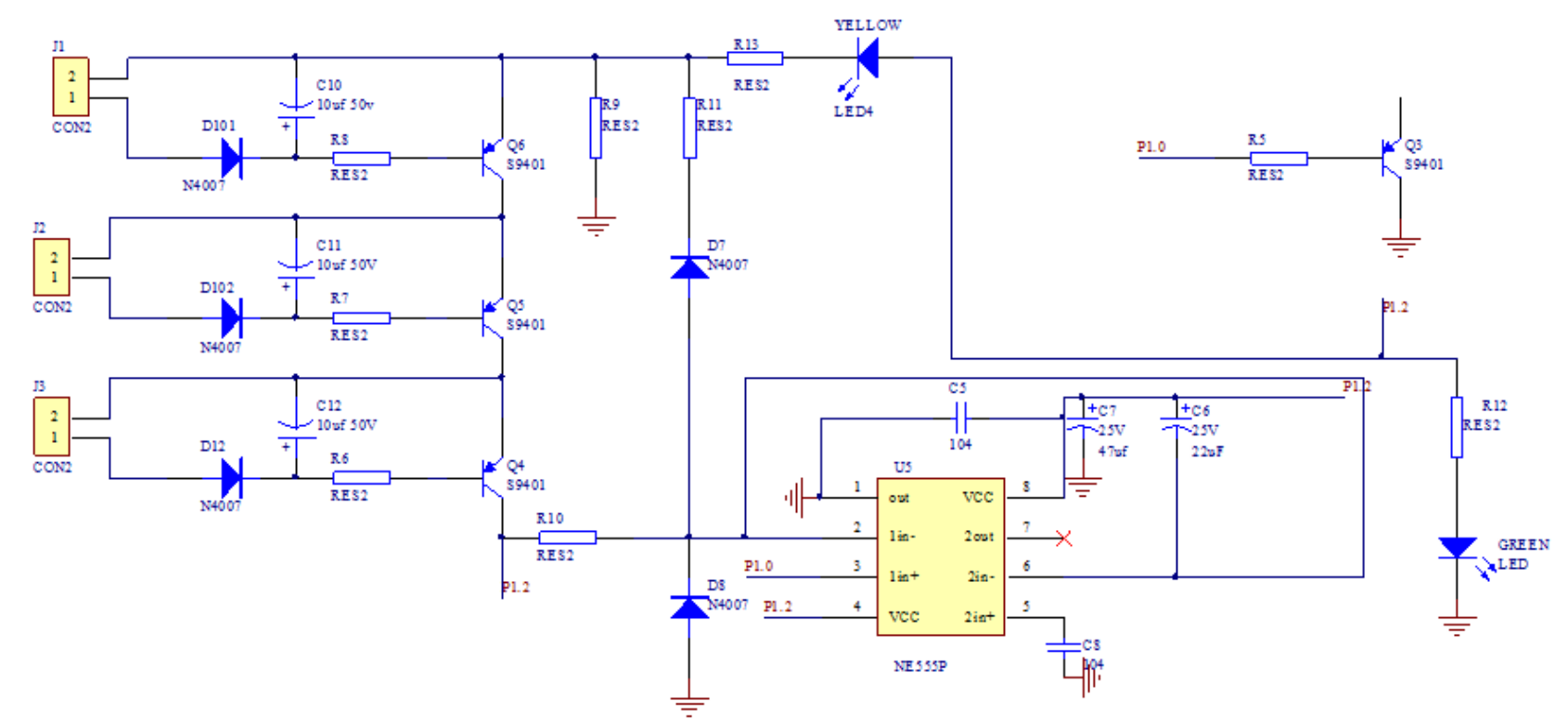

Fig. 5 Phase lack protection circuit

\section{Phase Lack Protection Circuit}

In the controlled circuit, phase lack protection circuit can prevent the motor of the rolling machine from destroying while three-phase motor lies in the missing phase operation. The following shows the basic principle of the circuit.

$\mathrm{J} 1, \mathrm{~J} 2$, and $\mathrm{J} 3$ represent three inductance coil set in two phase, respectively. When three-phase power lines are in good condition without self-induced electromotive force in three inductance coil, and P1.0 pin has low level. So the transistor Q3 lies in the conduction state, relay circuit work normally, and the AC contactor controlled by the relay circuit works well. When one of the three phase coils has no current, inductive electromotive force is produced between in the induction coil J1, J2 and J3, then the triodes Q4 - Q6 lies in the off state. The third pin P1.0 of the timer 555 outputs the high level which lets Q3 lie in the off state. So the relay works to shut off the main circuit power supply, and the motor can be protected.

\section{Conclusion}

This paper designs a new rolling machine intelligent control system for greenhouse based on IOT. This system with many modules uses CC2530 as the core. CC2530 compares the data from sensors with pre-set values from the mobile, computer or keyboard, considering the current time and date, to control the rolling machine automatically. The rolling machine can be controlled by manually, remotely, and automatically. In this paper, several important parts, including design schematic diagram and produces, have been shown. This system can save a lot of labours and let fruits and vegetables get suitable temperature and humidity. After a long work verification, the system has the performances of safety and reliability, low cost and can be embedded into agricultural IOT.

\section{Acknowledgments}

The authors wish to express their gratitude to the projects: Design of Standardized Breeding System for Rabbits Based on Internet of Things from Education Department of Jilin Province, Jilin Province Economic Structural Adjustment Leading Fund Special Project (No. 2014Y108) and Changchun City Science and Technology Plan Project (No. 14nk029, No. 13KG71), Key Tackling Item of Jilin Province Science \& Technology Department (No. 20140204045NY), Jilin Province Science \& Technology Department Project (No. 20150204058NY, No. 20140204045NY) for their generous support of this work. 


\section{References}

[1]. SUN Hao, TENG Guanghui, ZHANG Xiaofei, et al. Design and Test on Real-time Measurement System of Mat Roller Workload for Sunlight Greenhouse [J]. Beijing: Transactions of Chinese Society of Agricultural Engineering, 2014, 30 (1): 138-145, in Chinese.

[2]. SUN Guoli, SU Yu. Design on Automatic Rolling Machine and Intelligent Ventilation Control System of Sunlight Greenhouse [J]. Hubei: Hubei Agricultural Sciences, 2015, 54 (24): 6386-6388, 6393, in Chinese.

[3]. ZHANG Jie, LU Boyou, ZHANG Haihui, et al. Design of Precision Control System Based on ZigBee for Greenhouse Met Roller [J]. Heilongjiang: Journal of Agricultural Mechanization Research, 2013, (5): 77-80, in Chinese.

[4]. WANG Kaide, LI Penghui. The Modified Design of Control System Based on the Mobile Met Roller of Greenhouse [J]. Anhui: Journal of Anhui Agricultural Sciences, 2014, 42 (25): 8837-8839, in Chinese.

[5]. LIU Lin, HOU Jialin, LANG Xuqiang, et al. Design of Intelligent Rolling Machine of Solar Greenhouse [C]. 2012 Chinese Agricultural Machinery Association International Academic Conference, 2015: 69-73, in Chinese. 